\title{
Non-Hodgkin's lymphoma and spinal cord compression: a diagnostic challenge
}

\author{
Bilal Bin Abdullah, ${ }^{1}$ Nida Nausheen, ${ }^{2}$ Nagur Khuda Baksh, ${ }^{1}$ Naga Dharma Teja Keerthi ${ }^{1}$ \\ ${ }^{1}$ Department of Medicine; and 2Department of Pathology, Al Ameen Medical College Hospital, Bijapur, Karnataka, India
}

\begin{abstract}
We report a 25-year-old lady presenting to the outpatient department of a tertiary care hospital with spinal cord compression. Initially we had a diagnostic difficulty, as its presentation was perplexing. Finally we pointed towards the diagnosis of nonHodgkin's lymphoma and proved it. This article carries a message for the clinicians as clinical fallacies were noticed towards pointing this diagnosis. We have clearly explained the shortcomings in evaluating a case making a diagnosis towards nonHodgkin's lymphoma and spinal cord compression, as many situations in the setting were simulating and overlapping.
\end{abstract}

\section{Introduction}

Non-Hodgkin's lymphoma (NHL) is malignancy of lymphoid cells affecting multiple systems. Every year about 367,000 patients are diagnosed to have NHL. Extra nodal NHL constitutes about $10-20 \%$ of all NHL cases.

NHL accounts for more than $85 \%$ of cases of spine and epidural soft tissue involvement. It occurs between $40-60$ years of age. There is a strong male predominance. NHL can cause bone destruction and hyperostosis. Spinal cord compression occurs during the course of NHL in 0.1 to $6.5 \%$ of patients. Such involvement tends to develop late in the course of established disease when dissemination has occurred. Cord compression as the initial presenting feature of lymphoma is rare. Epidural extension is best delineated on magnetic resonance imaging (MRI).

Correspondence: Bilal Bin Abdullah, Department of Medicine, Al Ameen Medical College Hospital, Bijapur, Karnataka, India.

E-mail: drbilal28@yahoo.com

Key words: Non-Hodgkin's lymphoma; magnetic resonance imaging; lumbar vertebra.

Received for publication: 10 December 2014.

Revision received: 12 January 2015.

Accepted for publication: 4 February 2015.

This work is licensed under a Creative Commons Attribution NonCommercial 3.0 License (CC BY-NC 3.0).

CCopyright B.B. Abdullah et al., 2015

Licensee PAGEPress, Italy

Italian Journal of Medicine 2015; 9:380-383

doi:10.4081/itjm.2015.573
Spinal cord compression is a rare presentation of NHL occurring only in $0.1 \%$ to $3.3 \%$ of patients of primary extradural NHL. ${ }^{1}$ However, spinal cord compression may occur in $0.1 \%$ to $10.2 \%$ of NHL patients during the course of disease and is usually aggressive in behavior. ${ }^{2}$ It is most commonly caused by extradural disease, either due to an isolated deposit within the spinal canal or by extension from an adjacent nodal mass or bone involvement. ${ }^{3}$ NHL that occurs with spinal epidural involvement is often an aggressive disease. In contrast, primary spinal extradural NHL, i.e., occurring in the absence of any detectable extra spinal lymph proliferative disorder, has a potentially favorable outcome if diagnosed and treated early, ${ }^{4}$ the lesion being markedly sensitive to radiation and chemotherapy. The clinical features and outcome vary with the duration of symptoms, final diagnosis and surgical intervention. Patients presenting with spinal cord compression and no defined primary lesion elsewhere, require surgical intention for diagnosis and relief of neurological deficit. ${ }^{5}$ This varied presentation of NHL presents a diagnostic discrepancy with clinically simulating conditions as Pott's spine.

The other differentiating conditions are rhabdomyosarcomas, Ewing's sarcoma, vertebral metastasis from other known defined primary sites and in very rare situations NHL as from thyroid gland itself.

\section{Case Report}

A 25-year-old female presented to the outpatient department of a tertiary care hospital with symptoms of dull aching low backache increased with physical exertion and difficulty in walking since 2 months associated with tingling and numbness sensation in right lower limbs intensity of pain increased since last 10 days radiating to thighs, back and both her buttocks. 
The neurological disability noticed was that she has to walk with an aid. On a scale of 10, patient graded pain as 7. On examination patient's general condition was fair, no lymphadenopathy, no weight loss, weight was $59 \mathrm{~kg}$ and appetite was normal. Blood pressure $124 / 80 \mathrm{Hg}$, pulse rate 86 per minute, respiratory rate 20 per minute. Cardiovascular, respiratory and per abdominal examination was normal. Central nervous system examination showed decreased power in the lower limbs.

Both the lower limbs with power of grade $3 / 5$ in hip compartment, $4 / 5$ in flexors and extensors of knee. Deep tendon reflexes were diminished. Sensory system was intact with the rest of neurological examination being normal. A point of tenderness was noticed in lower back at $\mathrm{L} 3$ level a clinical diagnosis of spinal cord compression presenting as cauda equina syndrome was considered and investigated. White blood cells count was 11,000 cells per cu mm, erythrocyte sedimentation rate 85 , hemoglobin $12 \mathrm{gm}$ and peripheral smear was within normal limits. All other baseline investigations were within normal limits. Chest x-ray and abdominal ultrasound was normal. On the second day in hospital course she had severe backache radiating to thighs and graded it as 10 on a scale of 10 .

She underwent MRI spine which showed destruction of L3 vertebra with extensive soft tissue in anterior and posterior epidural spaces, compressing thecal sac. The lesion was hypo intense on T1 and hyper intense on T2 weighted image sequences. Lumber vertebra normal in alignment. Marrow of the rest of vertebra, vertebral disc and posterior vertebral appendages showed normal signal intensities. No event of abnormal signal intensities in the cord. MRI was suggestive of lesion causing destruction of L3 vertebra associated with extensive soft tissue in anterior and posterior epidural space seen compressing thecal sac (Figure 1).

Core biopsy was done from L3 additional cut sections were examined, the marrow was hyperplastic, one focus of marrow fibrosis seen with crushed dark cells. On deeper sections the cells appeared to be polymorphic with both small lymphocytes and few larger cells. A definitive opinion was not formed as small part of the lesion had come in to the biopsy tissue. In view of cellular polymorphism an inflammatory pathology was suggested and trial with anti-tubercular treatment was considered.

At this stage anti-tubercular treatment was initiated and patient was discharged home. She reported back to us within 5 days with severity in her symptoms and unable to walk. On examination power was grade $2 / 5$ in hip compartment. MRI was repeated and showed herniation of L2 L3 L4 intervertebral disc into centrum of adjacent end plates causing partial collapse with heterogeneous altered marrow signal involving the body as well as posterior elements of L3. The ad- jacent disc appeared normal, except for degeneration. Extensive abnormal epidural soft tissue at L3 level obliterating thecal sac involving L3 L4 neural foramina. The nerve roots of cauda equina are not visualized separately from the soft tissue associated with well illdefined abnormal soft tissue along the medial fiber of right psoas muscle at L2 to L4 level.

With this yet a possibility of Potts spine was considered and anti-tubercular treatment was continued, furthemore the orthopedic surgeon placed internal fixators for L3 L4 vertebras and patient underwent a bone scan.

Bone scan was done with 74 MBQ 99m Tc MPD administrated. Whole body skeletal survey done $3 \mathrm{~h}$ later. It showed abnormal uptake seen in L3 L4 vertebra. Left $\mathrm{S} 1$ joint showed abnormal accumulation in anterior view. Left hip joint (acetabulum) showed abnormal accumulation. Impression was multiple skeletal metastases in the above-mentioned areas. As this posed a diagnostic dilemma again a linear bone biopsy was repeated from L3 vertebra, which showed hyper cellular marrow. One focus of cohesive cells in the marrow of the nuclei was large and oval to round with vesicular chromatin. The cytoplasm was clear to fairly eosinophilic and granular suggestive of neoplastic deposit in the marrow. To further characterize these cells immunostaining for leukocyte common antigen was done which was negative thus excluding the possibility of lymphomatous deposit. There were no granulomas or giant cells. Impression was of metastatic carcinoma deposit in the marrow L3 level.

Work up for primary source, including thyroid was suggested. Thyroid scan was done with $111 \mathrm{MBq}$ of pertechnetate and was normal. Lactate dehydrogenase was 1560 and we have sent the same biopsy material for immune histochemistry, which confirmed the diagnosis of NHL B cell type. She underwent chemotherapy and radiation successfully. Presently she is doing her day-to-day activity.

\section{Discussion and Conclusions}

We describe NHL presenting with signs and symptoms of spinal cord compression cauda equina syndrome. MRI spine was suggestive of a tubercular process and was treated accordingly. We had a strong suspicion of an existing lymphoma and the core biopsy material obtained initially was less. The report remained inconclusive. Computed tomography (CT) of the thorax was performed mainly to look for any possibility of lymph nodes and mediastinum was viewed to see any existing nodes.

Abdominal CT scan was normal. There was no event of any lymphadenopathy or splenomegaly. On fifth day of discharge from the hospital she reported with severity in her symptoms, her power was grade $2 / 5$. An MRI scan was repeated which showed herni- 
ation in L2 L4. In the meantime an orthopedic surgeon intervened and placed her with internal fixators. A bone scan was carried out and was suggestive of skeletal metastasis of L3 L4 and acetabulum of hip.

A deep core biopsy was repeated and was suggestive of vertebral metastasis and leukocyte antigen was negative for lymphomas. A thyroid scan was done for possibility of lymphoma and was negative. So none of the investigations were pointing towards lymphoma.

As per the literature NHL involves paravertebral lymph nodes and causes adjacent vertebral invasion with or without intra-paraspinal involvement, it may also involve epidural space in isolation and may pres- ent as intra medullary mass. The MRI favors the diagnosis of tubercular process with soft tissue involvement. Here the vertebral body alignment was normal initially, pedicles were also normal and this MRI was not consistent to define the spinal occult NHL. Four patterns of vertebral metastases are seen on MRI focal lytic, focal sclerotic, diffuse homogenous, diffuse inhomogeneous. Lytic lesions are most common. They are hypo intense on $\mathrm{T} 1$ and hyper intense on $\mathrm{T} 2 \mathrm{~W}$ images. Sclerotic lesions are hypo intense on $\mathrm{T} 1$ and T2WI. Diffuse homogenous pattern involves multiple vertebrae, which are hypo intense on T1 and hyper intense/heterogeneous on T2W images. Diffuse heterogeneous pattern involves multiple vertebrae, which

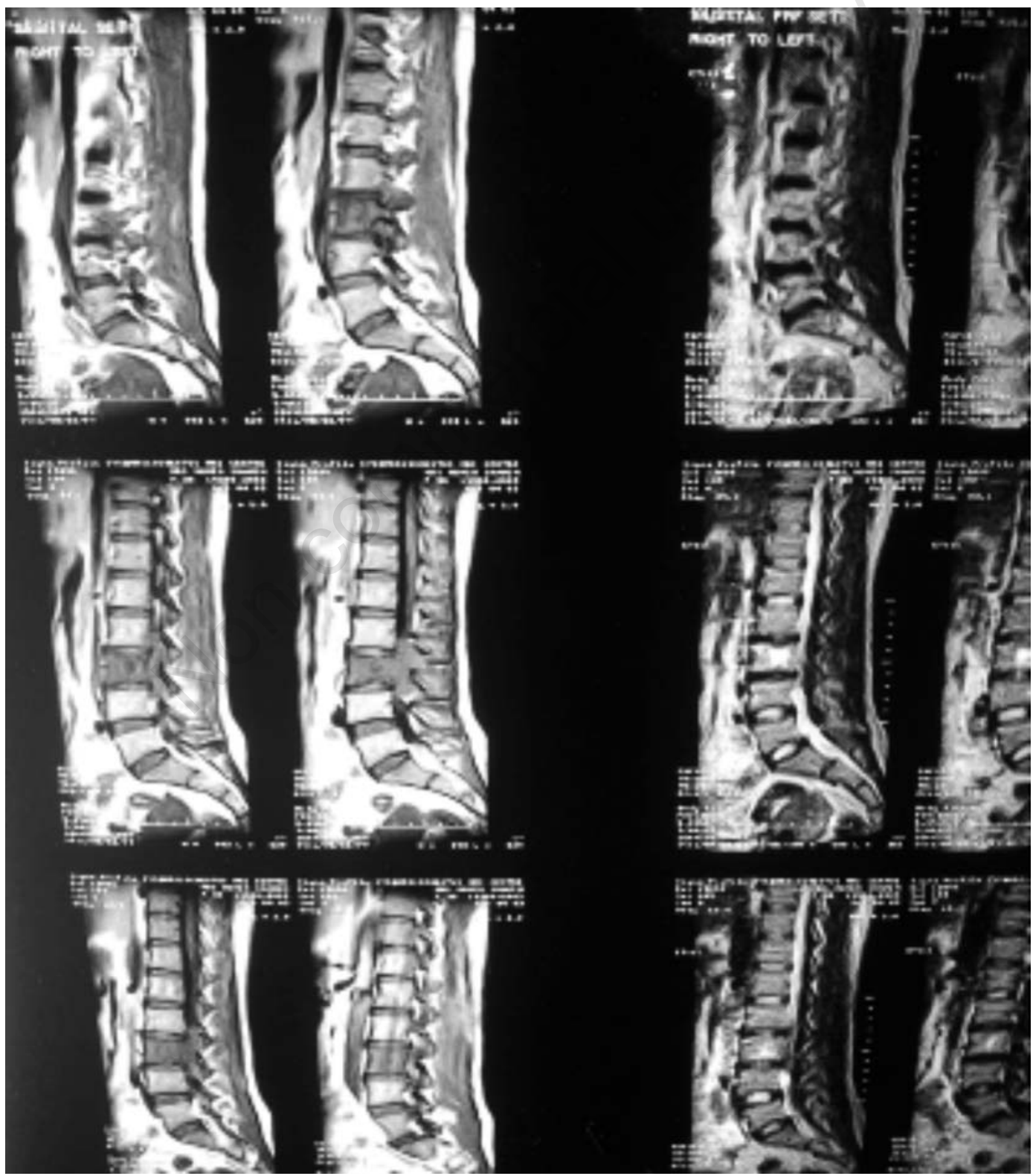

Figure 1. Magnetic resonance images of the spine. 
show mixed signal intensities on $\mathrm{T} 1$ and $\mathrm{T} 2 \mathrm{~W}$ images. The immunohistochemistry report was suggestive of NHL B type. She underwent treatment accordingly. We have clearly described the clinical situations simulating, overlapping likely to create a clinical fallacy. Authors have expressed the approach in distinguishing and differentiating each of these clinically confusing situations in setting of cord compression.

\section{References}

1. Freeman C, Bing JW, Cutter SJ. Occurrence and prognosis of extradural lymphoma. Cancer 1972;29:252-60.
2. Aabo K, Walbom Jorgenee S. Central versus system epicates by malignant lymphatic. Radiation schedule and treatment results. Int J Radiat Oncol Biol Phys 1986;12: 195-202.

3. Eeles RA, O'Brien P, Horwich A, Brada M. NonHodgkin's lymphoma presenting with extraduralspinal cord compression: functional outcome and survival. $\mathrm{Br}$ J Cancer 1991;63:126-9.

4. Epelbaum R, Haim N, Ben-Shahar M, et al. NonHodgkin's lymphoma presenting with spinal epidural involvement. Cancer 1986;58:2120-4.

5. Bragg DG, Cosby TV, Ward JH. New Concepts in the non-Hodgkin's lymphoma; radiologic implications. Radiology 1989;159:289-304. 